\title{
Structure of the Alkalohyperthermophilic Archaeoglobus fulgidus Lipase Contains a Unique C-Terminal Domain Essential for Long-Chain Substrate Binding
}

\author{
Cammy K.-M. Chen ${ }^{1,2} \dagger$, Guan-Chiun Lee ${ }^{3} \dagger$, Tzu-Ping Ko ${ }^{2}$, \\ Rey-Ting Guo ${ }^{2}$, Li-Min Huang ${ }^{4}$, Hsiao-Jung Liu ${ }^{3}$, Yi-Fang $\mathrm{Ho}^{4}$, \\ Jei-Fu Shaw ${ }^{4,5 *}$ and Andrew H.-J. Wang ${ }^{1,2,6 *}$
}

${ }^{1}$ Institute of Biochemical

Sciences, National Taizwan University, Taipei 106, Taiwan

${ }^{2}$ Institute of Biological Chemistry, Academia Sinica, Taipei 115, Taiwan

${ }^{3}$ Department of Life Science, National Taiwan Normal University, Taipei 106, Taiwan

${ }^{4}$ Institute of Plant and Microbial Biology, Academia Sinica, Taipei 115, Taiwan

${ }^{5}$ Department of Food Science and Biotechnology, National Chung Hsing University, Taichung 402, Taiwan

${ }^{6}$ Core Facility for Protein Crystallography, Academia Sinica, Taipei 115, Taiwan

Received 2 February 2009; received in revised form 27 April 2009; accepted 12 May 2009
Several crystal structures of AFL, a novel lipase from the archaeon Archaeoglobus fulgidus, complexed with various ligands, have been determined at about $1.8 \AA$ resolution. This enzyme has optimal activity in the temperature range of $70-90{ }^{\circ} \mathrm{C}$ and $\mathrm{pH} 10-11$. AFL consists of an Nterminal $\alpha / \beta$-hydrolase fold domain, a small lid domain, and a C-terminal $\beta$-barrel domain. The N-terminal catalytic domain consists of a 6-stranded $\beta$-sheet flanked by seven $\alpha$-helices, four on one side and three on the other side. The $C$-terminal lipid binding domain consists of a $\beta$-sheet of 14 strands and a substrate covering motif on top of the highly hydrophobic substrate binding site. The catalytic triad residues (Ser136, Asp163, and His210) and the residues forming the oxyanion hole (Leu31 and Met137) are in positions similar to those of other lipases. Long-chain lipid is located across the two domains in the AFL-substrate complex. Structural comparison of the catalytic domain of AFL with a homologous lipase from Bacillus subtilis reveals an opposite substrate binding orientation in the two enzymes. AFL has a higher preference toward long-chain substrates whose binding site is provided by a hydrophobic tunnel in the $\mathrm{C}$-terminal domain. The unusually large interacting surface area between the two domains may contribute to thermostability of the enzyme. Two amino acids, Asp61 and Lys101, are identified as hinge residues regulating movement of the lid domain. The hydrogen-bonding pattern associated with these two residues is $\mathrm{pH}$ dependent, which may account for the optimal enzyme activity at high $\mathrm{pH}$. Further engineering of this novel lipase with high temperature and alkaline stability will find its use in industrial applications.

(C) 2009 Published by Elsevier Ltd.

Keywords: lid; catalytic triad; interface; hinge; hydrophobic tunnel
${ }^{*}$ Corresponding authors. E-mail addresses:

presid@dragon.nchu.edu.tw; ahjwang@gate.sinica.edu.tw.

$\dagger$ C.K.-M.C. and G.-C.L. contributed equally to this work.

Abbreviations used: AFL, Archaeoglobusfulgidus lipase; MIR, multiple isomorphous replacement; PEG, polyethylene glycol; pNPP, $p$-nitrophenyl palmitate; BSL, Bacillus subtilis lipase; mAFL, mature AFL; tAFL, Cdomain-truncated AFL; pAFL, premature AFL; PL, pancreatic lipase; PDB, Protein Data Bank; TTR, transthyretin.

\section{Introduction}

Since the beginning of the 1990s, the crystal structures of several lipases have been determined. ${ }^{1,2}$ The structures have attracted much interest because these enzymes can be used as biocatalysts not only for the hydrolysis of fats and oils but also for the synthesis of a large number of unnatural products. ${ }^{3}$ The enzymes are frequently used for industrial-scale production of fine chemicals and pharmaceuticals. ${ }^{3}$ 75 
Lipases are versatile in functions. In addition to their natural function of hydrolyzing carboxylic ester bonds, they also catalyze esterification, interesterification, and transesterification reactions in nonaqueous media. Such versatility makes them useful for a wide range of applications in the food, detergent, leather, pharmaceutical, textile, cosmetic, and paper industries. ${ }^{4}$

Lipases (EC 3.1.1.3) are triacylglycerol ester hydrolases that catalyze the hydrolysis of longchain acylglycerols. Lipases and carboxylesterases differ in their substrate preferences: carboxylesterases prefer hydrophilic substrates whereas lipases favor long-chain hydrophobic fatty acid esters. Lipases generally have a broad substrate spectrum, though sometimes they possess a remarkable regioselectivity and enantioselectivity. ${ }^{5}$ Lipases are widely distributed throughout the plant and animal kingdoms, as well as in molds and bacteria. Although lipases vary considerably in size, they all presumably have a similar $\alpha / \beta$-hydrolase fold embedded with a catalytic triad consisting of serine, histidine, and aspartate (or glutamate). The serine residue is located in a strictly conserved $\beta$ Ser- $\alpha$ motif. The active site of lipase is often covered by an amphipathic helical lid domain, which moves away upon contact of the lipase with its substrate, thereby exposing hydrophobic binding pocket at the protein's surface. ${ }^{2}$ In the presence of waterlipid interface, the lid opens and the enzyme activity is increased, a phenomenon called interfacial activation. $^{6}$

Bacterial lipases have recently been classified into eight different families, with family I being the largest and consisting of six subfamilies. ${ }^{7}$ These lipases usually show pronounced differences in regioselectivity and enantioselectivity despite a high degree of amino acid sequence homology.2,7-10 Archaeoglobus fulgidus archaeabacteria are hyperthermophilic marine sulfate reducers found in hydrothermal environments. They were isolated from hot oil field waters from an oil production platform. They are anaerobes that utilize lactate, pyruvate, and valerate plus $\mathrm{H}_{2}$ as carbon and energy sources with sulfate as electron acceptor. ${ }^{11}$ Studies on lipases from hyperthermophilic bacteria such as A. fulgidus lipase (AFL) should provide additional insights into the identification of the structural elements important to protein's thermostability, while providing useful information for future applications in biocatalysis and biotechnology. ${ }^{12,13}$

The AFL is particularly interesting, since it not only is hyperthermophilic and alkalophilic but also contains a unique $\mathrm{C}$-terminal domain. This enzyme showed optimal activity at $70-90{ }^{\circ} \mathrm{C}$ and between $\mathrm{pH} 10$ and 11, which is among the most alkaline $\mathrm{pH}$ range detected for hydrolases. ${ }^{14,15}$ Previously, the AFL gene was overexpressed in Escherichia coli, purified, and characterized. ${ }^{14}$ On the basis of a secondary-structure-driven multisequence alignment, the N-terminal half of AFL was predicted to adopt the hydrolase topological fold and possess catalytic activity since a truncated version covering the $244 \mathrm{~N}$-terminal residues retained measurable 139 activity. ${ }^{14}$ The function of the C-terminal domain in 140 AFL was not clear. The large amounts of biochem- 141 ical data and many crystal structures of lipases 142 enabled good understanding of their catalytic 143 mechanism, reaction selectivity, and substrate spe- 144 cificity, but none of the known bacterial lipase 145 structures contains an extra C-terminal domain for 146 long-chain substrate binding. ${ }^{16-19}$ In the present ${ }_{147}$ work, we report the crystallographic analysis on a 148 newly purified recombinant lipase cloned from the 149 hyperthermophilic archaeon A. fulgidus, which was 150 overexpressed in E. coli. The crystal structure of AFL 151 at $\sim 1.8 \AA$ resolution showed that the AFL is the first 152 lipase structure reported to contain a unique C- 153 terminal domain that is essential for binding long- 154 chain substrate.

\section{Results and Discussion}

\section{X-ray structure determination}

The structure of the enzyme was solved by 158 multiple isomorphous replacement (MIR) method, 159 and the refinement was first carried out using the 160 1.83-A-resolution data of the S136A mutant (Supple- 161 mentary Table 1). The structures of native AFL were 162 determined in three different crystals, obtained by 163 using isopropanol, polyethylene glycol (PEG) 4000, 164 and PEG 8000 as the major precipitants and refined 165 to $2.30,1.77$, and $3.10 \AA$ resolution, respectively, all 166 yielding low $R$ and $R_{\text {free }}$ values and stereochemical $\quad 167$ deviations (Table 1). There are two AFL monomers 168 (A and B) in an asymmetric unit of the structures 169 crystallized using isopropanol and PEG 4000, both 170 in the space group $P 22_{1} 2_{1}$ (Forms I and II). How- 171 ever, one monomer per asymmetric unit is observed 172 in the structure crystallized using PEG 8000 in the 173 space group $\mathrm{P}_{3}$ (Form III). The mutant S136A is 174 also crystallized from isopropanol in the Form I 175 unit cell.

The C-terminal His tag was not observed in any of 177 the seven crystallographically independent mono- 178 mer structures. When these seven independent AFL 179 monomers are superimposed using the program 180 $\mathrm{sPDBV}^{20}$ the root-mean-square deviations are $0.43-181$ $0.64 \AA$. In general, the respective N-terminal domain 182 and the C-terminal domain superimpose well, 183 except the lid region on top of the N-terminal 184 domain (Supplementary Fig. 1a). Although the 185 conformational change could be a result of crystal 186 packing, it may also represent flexibility of the lid 187 domain. In the AFL structures determined under 188 three different crystallization conditions, we 189 observed the binding of one $\mathrm{Ca}^{2+}$, three $\mathrm{Mg}^{2+}$, and 190 one $\mathrm{Cl}^{-}$. When these three structures are super- 191 imposed, we see the different ions all bind to the 192 position around the top of the C-terminal domain, 193 suggesting that these ions may help stabilize the 194 structure of the C-terminal domain (Supplementary Fig. 1b).

9
0
2
3
4
6
7
8
9
150
15
153
54


t1.2 Table 1. Data collection and refinement statistics for AFL crystals

\begin{tabular}{|c|c|c|c|c|c|}
\hline $\mathrm{t} 1.3$ & Names & $\begin{array}{c}\text { Native (isopropanol) } \\
\text { [Form I] }\end{array}$ & $\begin{array}{c}\mathrm{S} 136 \mathrm{~A}+p \mathrm{NPP} \\
{[\text { Form I] }}\end{array}$ & $\begin{array}{c}\text { Native (PEG 4000) } \\
{[\text { Form II] }}\end{array}$ & $\begin{array}{c}\text { Native (PEG 8000) } \\
\text { [Form III] }\end{array}$ \\
\hline $\mathrm{t} 1.4$ & \multicolumn{5}{|l|}{ Data collection } \\
\hline $\mathrm{t} 1.5$ & Space group & $P 2_{1} 2_{1} 2_{1}$ & $P 2_{1} 2_{1} 2_{1}$ & $P 2{ }_{1} 2_{1} 2_{1}$ & $\mathrm{P}_{3}$ \\
\hline $\mathrm{t} 1.6$ & Resolution $(\AA)^{\mathrm{a}}$ & $30-2.30(2.38-2.30)$ & $30-1.83$ (1.90-1.83) & $30-1.77$ (1.83-1.77) & $50-3.1(3.21-3.10)$ \\
\hline $\mathrm{t} 1.7$ & \multicolumn{5}{|l|}{ Unit cell dimensions $(\AA)$} \\
\hline $\mathrm{t} 1.8$ & $a$ & 89.44 & 91.20 & 52.29 & 99.55 \\
\hline $\mathrm{t} 1.9$ & $b$ & 105.38 & 107.87 & 106.67 & 99.55 \\
\hline $\mathrm{t} 1.10$ & $c$ & 117.00 & 118.90 & 175.89 & 59.02 \\
\hline $\mathrm{t} 1.11$ & \multicolumn{5}{|l|}{ No. of reflections } \\
\hline $\mathrm{t} 1.12$ & Observed & $276,752(24,748)$ & $605,017(54,461)$ & $346,404(27,062)$ & $77,200(7272)$ \\
\hline $\mathrm{t} 1.13$ & Unique & $50,018(4866)$ & $104,198(10,255)$ & $94,247(8682)$ & $10,557(1031)$ \\
\hline $\mathrm{t} 1.14$ & Completeness (\%) & $98.6(97.6)$ & $99.8(99.9)$ & $97.9(91.8)$ & $99.8(100.0)$ \\
\hline $\mathrm{t} 1.15$ & $R_{\text {merge }}(\%)$ & $6.0(27.2)$ & $5.1(42.9)$ & $6.5(34.4)$ & $6.2(40.0)$ \\
\hline $\mathrm{t} 1.16$ & $I / \sigma(I)$ & $37.0(8.1)$ & $31.8(5.4)$ & $23.9(2.4)$ & $35.8(5.0)$ \\
\hline $\mathrm{t} 1.17$ & & & & & \\
\hline $\mathrm{t} 1.18$ & Refinement & & & & \\
\hline $\mathrm{t} 1.19$ & No. of reflections & $47,885(4537)$ & $98,823(9164)$ & $90,064(7267)$ & $10,287(842)$ \\
\hline $\mathrm{t} 1.20$ & $R_{\text {work }}(95 \%$ data $)$ & $0.182(0.221)$ & $0.187(0.247)$ & $0.178(0.251)$ & $0.184(0.348)$ \\
\hline $\mathrm{t} 1.21$ & $R_{\text {free }}(5 \%$ data $)$ & $0.238(0.270)$ & $0.223(0.282)$ & $0.214(0.278)$ & $0.252(0.457)$ \\
\hline $\mathrm{t} 1.22$ & Geometry deviations & & & & \\
\hline $\mathrm{t} 1.23$ & Bond lengths $(\AA)$ & 0.017 & 0.020 & 0.019 & 0.014 \\
\hline $\mathrm{t} 1.24$ & Bond angles $\left(^{\circ}\right)$ & 1.8 & 1.8 & 1.9 & 1.9 \\
\hline $\mathrm{t} 1.25$ & No. of protein atoms & 7126 & 7202 & 7246 & 3632 \\
\hline $\mathrm{t} 1.26$ & Mean $B$ values $\left(\AA^{2}\right)$ & 27.7 & 26.2 & 27.5 & 67.3 \\
\hline $\mathrm{t} 1.27$ & No. of ligand atoms & 38 & 32 & 29 & 13 \\
\hline $\mathrm{t} 1.28$ & Mean $B$ values $\left(\AA^{2}\right)$ & 36.1 & 35.2 & 39.4 & 55.9 \\
\hline $\mathrm{t} 1.29$ & No. of ions & 2 & 2 & 5 & 1 \\
\hline $\mathrm{t} 1.30$ & Mean $B$ values $\left(\AA^{2}\right)$ & 20.5 & 19.0 & 37.6 & 46.6 \\
\hline $\mathrm{t} 1.31$ & No. of water molecules & 782 & 1226 & 1255 & 214 \\
\hline $\mathrm{t} 1.32$ & Mean $B$ values $\left(\AA^{2}\right)$ & 42.1 & 40.9 & 48.2 & 61.8 \\
\hline $\mathrm{t} 1.33$ & Ramachandran plot (\%) & & & & \\
\hline $\mathrm{t} 1.34$ & Most favored & 89.2 & 89.0 & 90.3 & 80.2 \\
\hline $\mathrm{t} 1.35$ & Additionally allowed & 10.3 & 10.5 & 9.2 & 19.3 \\
\hline $\mathrm{t} 1.36$ & Other & 0.5 & 0.5 & 0.5 & 0.5 \\
\hline
\end{tabular}

\section{Overall structure of AFL}

The overall structure shows a clear bipartite architecture composed of an N-terminal domain and a C-terminal domain. The $\mathrm{N}$-terminal domain (residues 1-237), which has an $\alpha / \beta$-hydrolase fold, carries the active site, while the $C$-terminal domain (residues 238-474) forms a $\beta$-barrel structure and provides the substrate hydrocarbon tail binding site (Fig. 1a). The N-terminal domain shows a single compact domain that consists of six parallel $\beta$ strands forming a $\beta$-sheet, sandwiched by seven $\alpha$ helices, with four helices on one side and three on the other side. The fold of the $\mathrm{N}$-terminal domain of AFL resembles that of the core of the $\alpha / \beta$-hydrolase enzymes, which is also seen in other lipases of known 3D structures. Lipase was traditionally believed to have a lid structure. By solving the crystal structure, we discovered the presence of a lid in the catalytic N-terminal domain to regulate the binding of the substrate. The AFL lid is composed of three helices, $\alpha 3, \alpha 4$, and $\alpha 5$ (residues 62-101), and two hinge residues, Asp61 and Lys101 (Fig. $1 \mathrm{a})$, and it adopts a closed conformation with clear electron densities, suggesting a well-ordered structure. The active site is located at the bottom of a hydrophobic crevice covered by the lid. Normally, the lid and the hinges are associated with the interfacial activation of lipases, where the $\alpha$-helical 224 lid opens up by rotating the two connecting hinge 225 regions.

The C-terminal domain (residues 238-474) is of 227 the $\beta$-sandwich type and is formed by two layers of 228 seven $\beta$-strands. The front antiparallel $\beta$-sheet 229 consists of strands $G$ to $M$, whereas the back $\beta-230$ sheet is made up of strands $\mathrm{N}$ to $\mathrm{T}$. At the C- 231 terminus, a substrate covering motif on top of the $\beta-232$ sandwich, composed of $\alpha 12, \alpha 13, \alpha 14$, and $\alpha 15,233$ forms a part of the hydrophobic substrate binding 234 tunnel. Since the substrate covering motif does not 235 cover the active site of AFL and no hinge can be 236 identified, it cannot be recognized as another lid. 237 The topology of AFL is shown in Supplementary 238 Fig. 1c. The native structure of AFL turns out to be a 239 fatty-acid-bound structure and this might be caused 240 by the binding of the protein to some fatty acid 241 fragments during its purification process. Since the 242 different AFL structures we obtained seem to bind 243 different lengths of fatty acid fragment, we suspect 244 that the fatty acid might be further extended at the 245 C-terminal end, reflecting multiple modes of bind- 246 ing. In the mutant structure of AFL (S136A) soaked 247 with $p$-nitrophenyl palmitate (pNPP), we still see the 248 binding of the fatty acid fragment. This might be 249 because the highly hydrophobic tunnel in AFL binds 250 to the fatty acid fragments tightly and prevents it 251 
252 from binding to other substrates such as pNPP.

253 Alternatively, the pNPP head group is slowly

254 hydrolyzed by the S136A mutant, which still

255 possessed very low activity.

\section{Comparison with Bacillus subtilis lipase}

Comparisons of known crystal structures of 257 lipases show that AFL has a similar catalytic 258

(a)
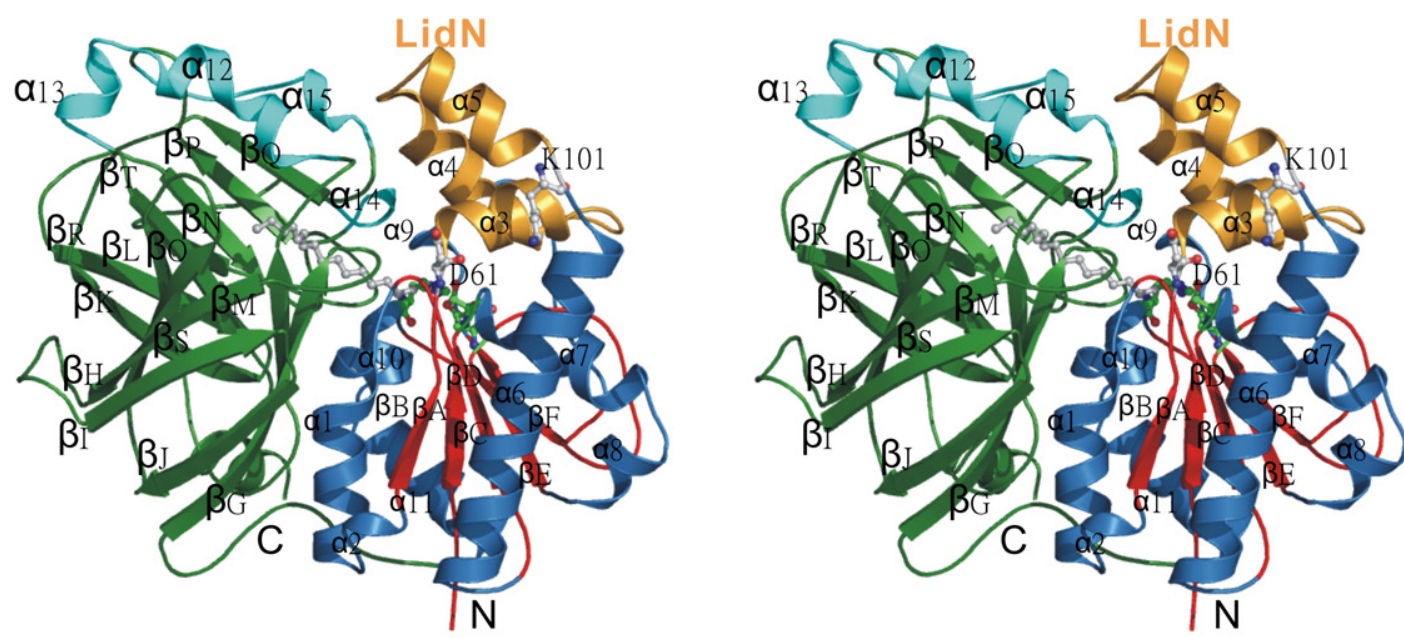

(b)
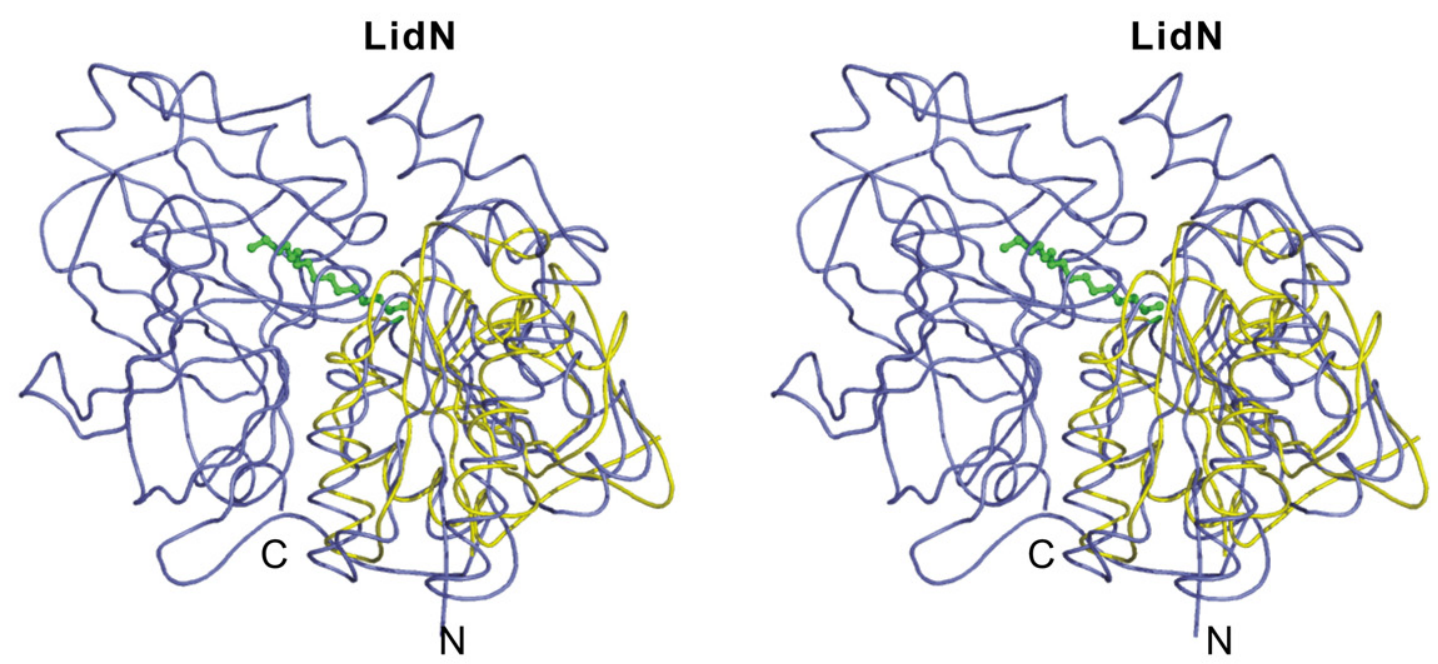

(c)

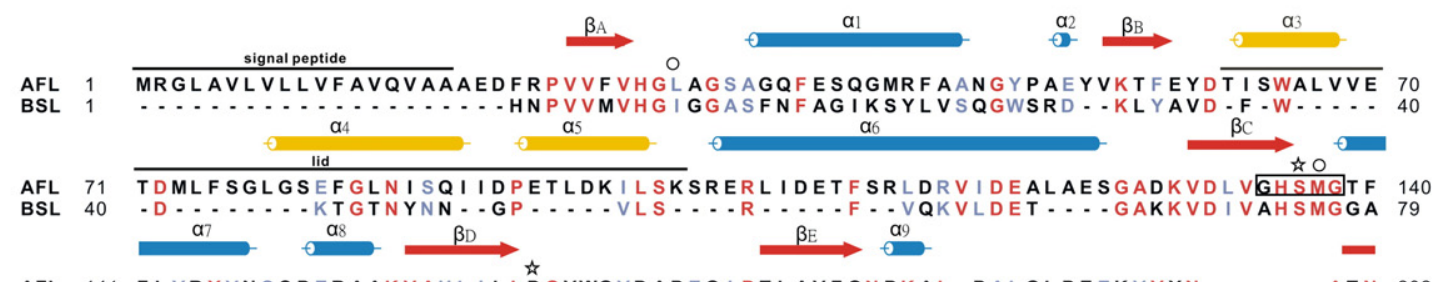

AFL 141 FLVRYVNSSPERAAKVAHLILLDGVWGVDAPEGIPTLAVFGNPKAL - PALGLPEEKVVYN . . . . - ATN 202 BSL 80 NTLYYIKNL - DGGNKVANVVTLGGANRLTTGKALP. -GTDPNQKILYTSIYSSADMIVMNYLSLDGARN 146

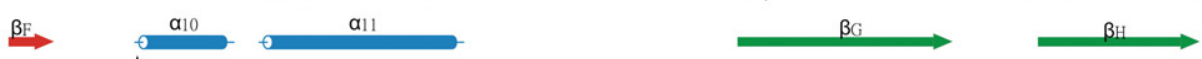

AFL 203 VYFNNMTH

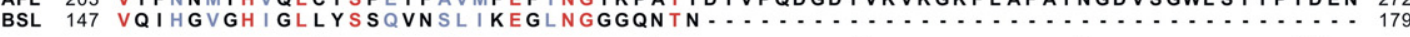

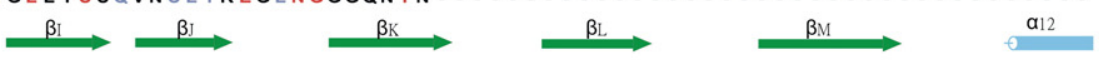

AFL 273 GKRLTRLPVKFMRVKGDFEVRLRKGQLYEFQFRKDFSPIIYHYYRAPFVRDDLWARFLVSKPPLDVELLI 342 $\stackrel{\alpha_{N}}{\longrightarrow} \stackrel{\alpha_{0}}{\longrightarrow}$

AFL 343 LPERLSPAAKETSGLLLIRYKEMIGEYDEEIGGVDEVYVNGVNVCTERICPIERAVNGLWVFDRGADGKS 412

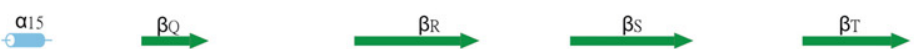

AFL 413 DLDREVVRYS IMPFMSAADLVVPAEGTISIAVKSRTGGEESFTIPAWSADRHSIIVQFSDYI 474

Fig. 1 (legend on next page) 
(a)
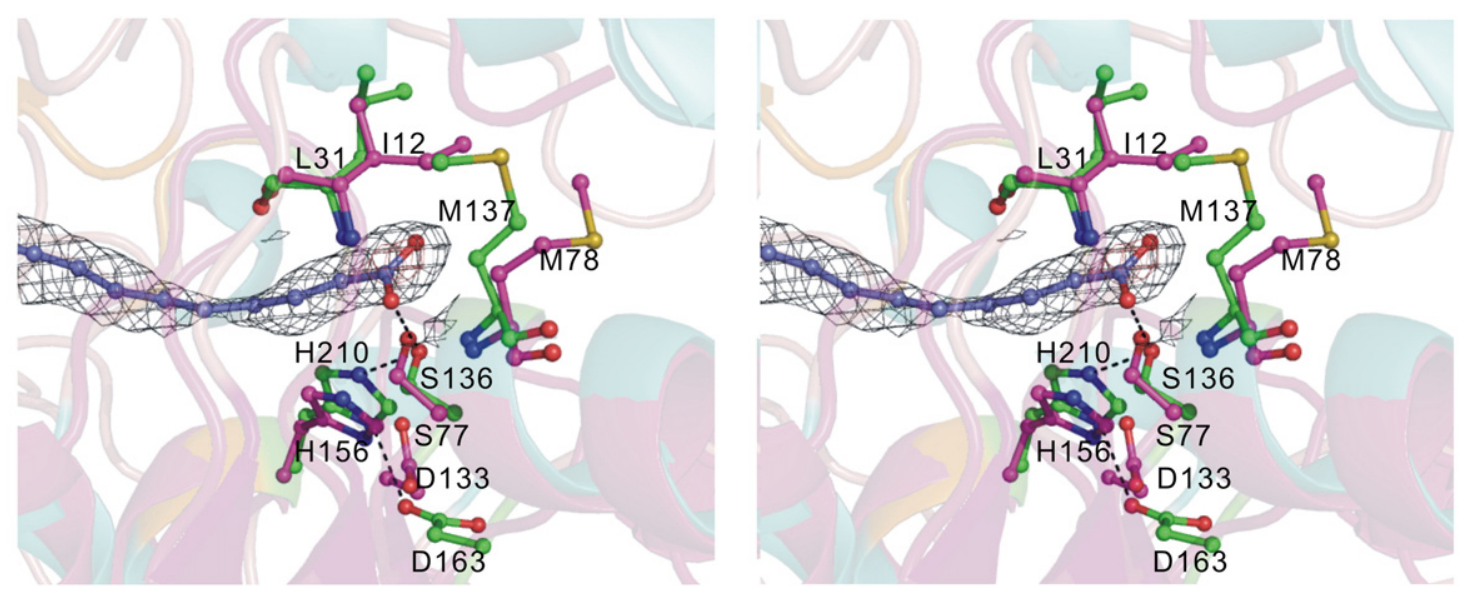

(b)
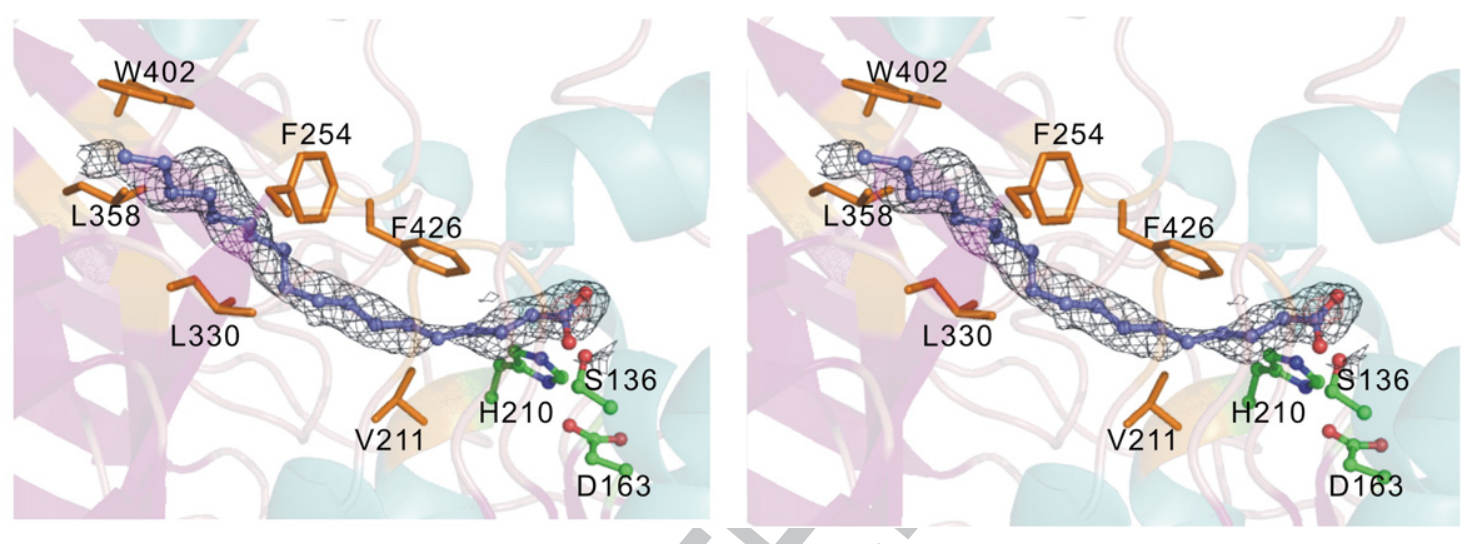

Fig. 2. Details of the interaction of AFL with its substrate in the active site. (a) A stereo view of the superimposition of the catalytic region of AFL and BSL. The figure shows the comparison of positions of the catalytic triad and oxyanion hole between the structures AFL (olive green) and BSL (purple). The AFL substrate is shown in blue as ball-and-stick structures. The $2 F_{\mathrm{o}}-F_{\mathrm{c}}$ electron density maps are contoured at $1 \sigma$ for the AFL substrate. Hydrogen bonds between the triad of AFL and the substrate are depicted as black broken lines. (b) A stereo view of the substrate binding tunnel in AFL. The substrate is shown in blue as ball-and-stick structures, and the $2 F_{\mathrm{o}}-F_{\mathrm{c}}$ electron density maps are contoured at $1 \sigma$ for the substrate. The side chains of the residues interacting with the substrate are shown as thicker lines (orange). The catalytic triad residues Ser136, Asp163, and His210 are shown in green as ball-and-stick structures.

domain. Further structural similarity search using DALI found that the structure of the B. subtilis lipase (BSL) exhibits the highest similarity to the Nterminal domain of AFL with a $Z$ score of 19.7. BSL also shows the highest similarity (42\%) and identity (23\%) to AFL. They can be superimposed with an rmsd of $1.25 \AA$ using 126 corresponding $\mathrm{C}^{\alpha}$ atoms. However, the BSL structure lacks not only the C-terminal domain shown in the AFL structure but also a lid. The comparison of BSL and AFL shows that AFL lacks an $\alpha$-helix between $\beta D$ and $\beta E$ and that it contains two additional $\alpha$-helices $\alpha 2$ and 270 $\alpha 8$ (Fig. 1b). Figure 1c shows the structure-based 271 sequence alignment of BSL and AFL, revealing that 272 the AFL structure contained an additional C- 273 terminal domain and a lid domain.

\section{Active site}

Based on the sequence alignment with BSL, the amino acids Ser136, His210, and Asp163 or Asp169 of AFL were assumed to be part of the catalytic

Fig. 1. The overall structure of AFL and BSL and the comparison between them. (a) A stereo view of the overall structure of AFL. The N-terminal domain contains six parallel $\beta$-strands, forming a $\beta$-sheet (red), and are sandwiched by seven $\alpha$ helices (blue) and a lid domain (orange). The C-terminal domain contains 14 antiparallel $\beta$-sheets (green) and a substrate covering domain (cyan). The substrate is shown in gray as a ball-and-stick model. The two hinge residues Asp61 and Lys101 are shown in gray as a ball-and-stick model. The catalytic triad is shown in green. (b) A stereo view of the superimposition of the AFL structure with BSL. The BSL structure (yellow) (PDB accession number 1r4z) shows a high degree of structural homology to the N-terminal $\alpha / \beta$-hydrolase fold domain of AFL (blue). (c) Structure-based sequence alignment of AFL and BSL (PDB accession number 1r4z). The secondary structure of AFL is shown above the alignment [colored as in (a)]. $\beta$-Strands are shown as arrows and $\alpha$-helices are shown as tubes. The numbering of all residues of the two proteins is shown on both sides of each line. Identical and similar amino acid residues are shown in red and sky blue, respectively. The lid and the signal peptide region are indicated. The stars indicate the catalytic triad residues, the circles indicate the oxyanion hole residues, and the box denotes the conserved GXSXG motif. The truncation site is shown with a pink arrow. 
triad. ${ }^{14}$ Here, we revealed that the catalytic triad residues Ser136 (as a nucleophile), Asp163, and His210 are actually located at their canonical positions in the $\alpha / \beta$-hydrolase fold. ${ }^{21}$ Their positions and orientations are similar to the catalytic triad residues in BSL except that the position of Asp163 has a small deviation from Asp133 of BSL (Fig. 2a). This is true also for the oxyanion hole, which is formed by the backbone nitrogen atoms of the residue immediately following the nucleophile and a residue located between strand $\beta \mathrm{A}$ and helix $\alpha 1$. In AFL, the oxyanion hole is formed by the peptide NH moieties of Leu31 and Met137, which are in a conserved position as the oxyanion hole (formed by Ile12 and Met78) of BSL (Fig. 2a). This shows that AFL has a preformed oxyanion hole similar to that of BSL. The nucleophilic Ser136, which was covered by the lid in the closed form of $\mathrm{AFL}$, is situated at the very sharp "nucleophile elbow", between strand $\beta C$ and helix $\alpha 7$. The catalytic serine is in an $\varepsilon$ characteristic conformation and is located in a tight turn with the G-H-S-M-G sequence belonging to the usual consensus sequence of the $\alpha / \beta$-hydrolase fold family.

\section{Acyl-binding site}

The active-site nucleophile Ser136 lies at the entrance of a hydrophobic tunnel. The tunnel consists of a deep canyon $20 \AA$ long and $7 \AA$ wide (Fig. 2b). The cavity is formed in AFL between the $\mathrm{N}$-terminal catalytic domain and the C-domain. Most residues of the tunnel wall are hydrophobic, the majority being leucines, phenylalanines, and valines (Fig. $2 b$ ). The structure of AFL with the fatty acid fragments shows the long-chain substrate fits in a hydrophobic tunnel lined by Val211, Phe254, Leu330, Leu358, Trp402, and Phe426. There is space for about 18 hydrocarbon units to be 315 accommodated in this tunnel.

The two hinge residues Asp61 and Lys101 at two 317 ends of the lid are located at the base of the lid 318 domain. The opening of the lid may be induced by 319 the hinge exerting a bending motion of the helical 320 lid, thereby creating an increased solvent-exposed 321 hydrophobic surface. By comparing the hinge points 322 of the four structures of AFL we obtained, a $\mathrm{pH}-323$ dependent conformational change at Lys101 was 324 observed (Fig. 3a). Among the four structures, the 325 conformation of Asp61 remains the same, but 326 Lys101 of the structures determined at different 327 $\mathrm{pH}$ values shows a different conformation. Detailed 328 investigation on the interaction of the two hinge 329 points shows that Lys101 interacts with Asp61 via 330 hydrogen bonding with Ser64 under a more acidic 331 crystallization condition (crystallized using isopro- 332 panol as the main precipitant, $\mathrm{pH}$ 4.6). However, 333 Lys101 undergoes a $90^{\circ}$ rotation to form a hydrogen 334 bond with Glu109 in a more basic crystallization 335 condition (crystallized using PEG 4000 and PEG 336 8000 as the main precipitant, $\mathrm{pH} 8.5$ and 10.5, 337 respectively) (Fig. 3b). It is reasonable to expect that 338 under a more basic condition, the conformational 339 change of Lys101 would render AFL to be more 340 readily to open and bind to its substrate. The 341 interaction of Lys101 with Glu109 instead of Ser64 342 probably enables the lid region in AFL to be more 343 flexible and have the ability to exert a larger extent of 344 movement. This might be the reason for AFL's 345 alkalophilic property.

The substrate binding tunnel is covered by the lid 347 domain and a substrate covering motif that lies 348 directly on top of the hydrophobic tunnel (Fig. 4a). It 349 is interesting to note that the four $\alpha$-helices on the 350 top of the C-terminal domain lie directly on the top 351 of the substrate binding site and block the contact 352 (a)

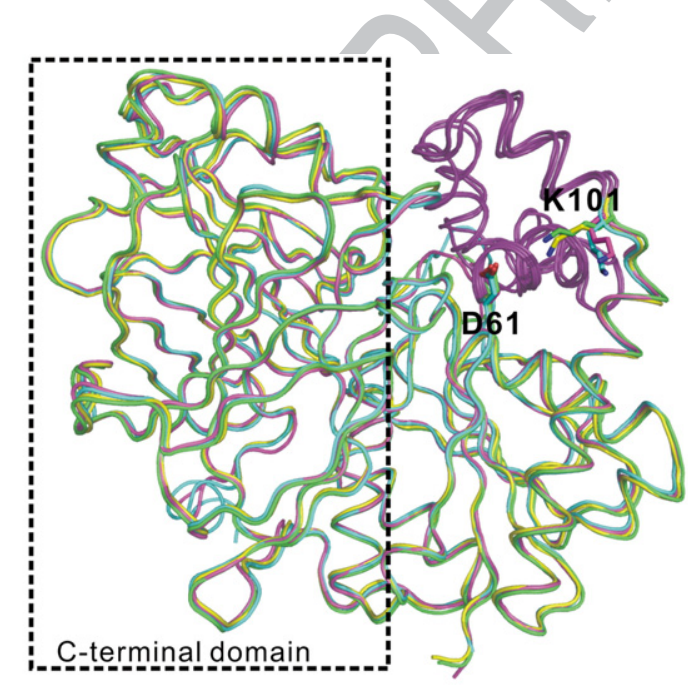

(b)

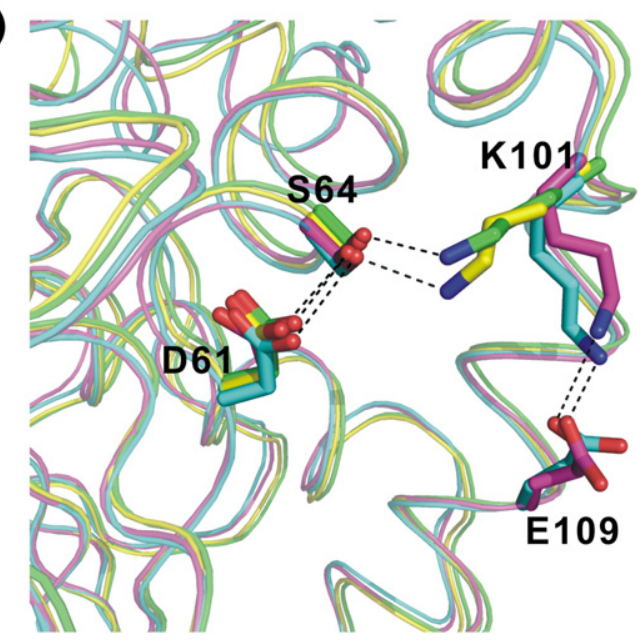

Fig. 3. The $\mathrm{pH}$-dependent conformational change of the hinge residues. (a) Superimposition of the subunits of native AFL (isopropanol) (yellow), mutant AFL S136A (isopropanol) (green), native AFL (PEG 4000) (cyan), and native AFL (PEG 8000) (magenta). The lid domain is shown as a purple loop. The hinge residues Asp61 and Lys101 of each of the four AFL structures are shown as sticks. (b) Details of the hinge residues. The hinge residues and the interacting residues Ser64, Asp61, and Glu109 of each of the four AFL structures are shown as sticks. Hydrogen bonds between the hinge residues and the interacting residues are depicted as black broken lines. 
(a)
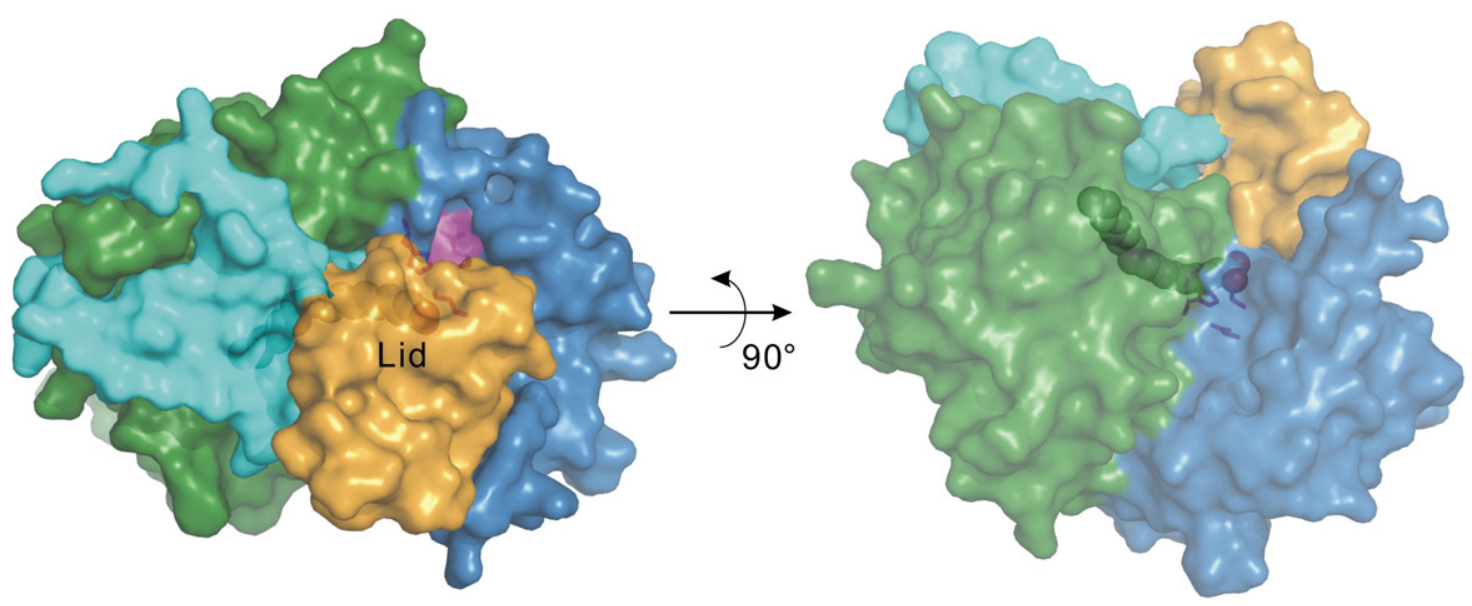

(b)

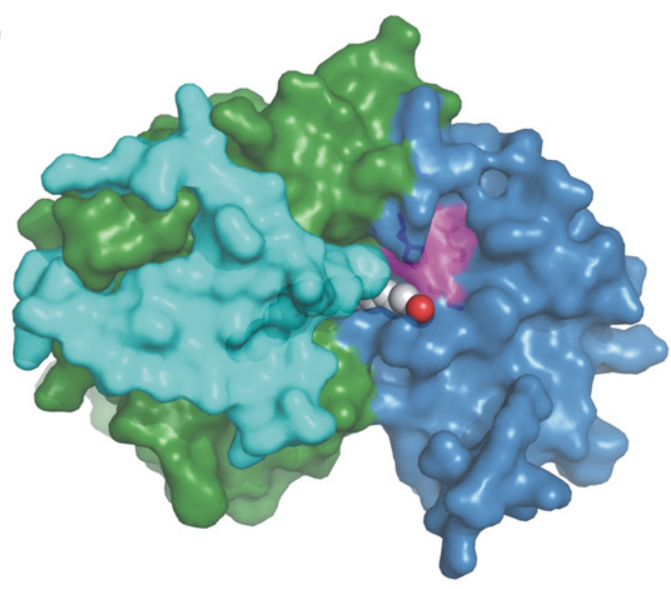

(c)

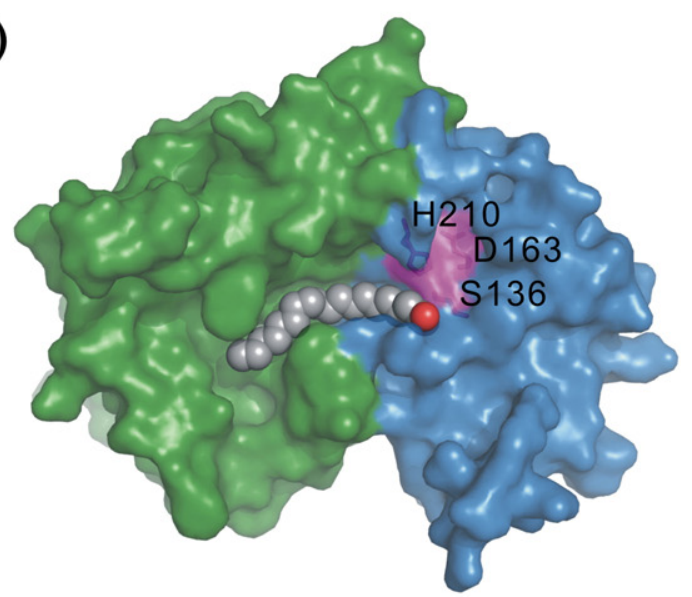

(d)
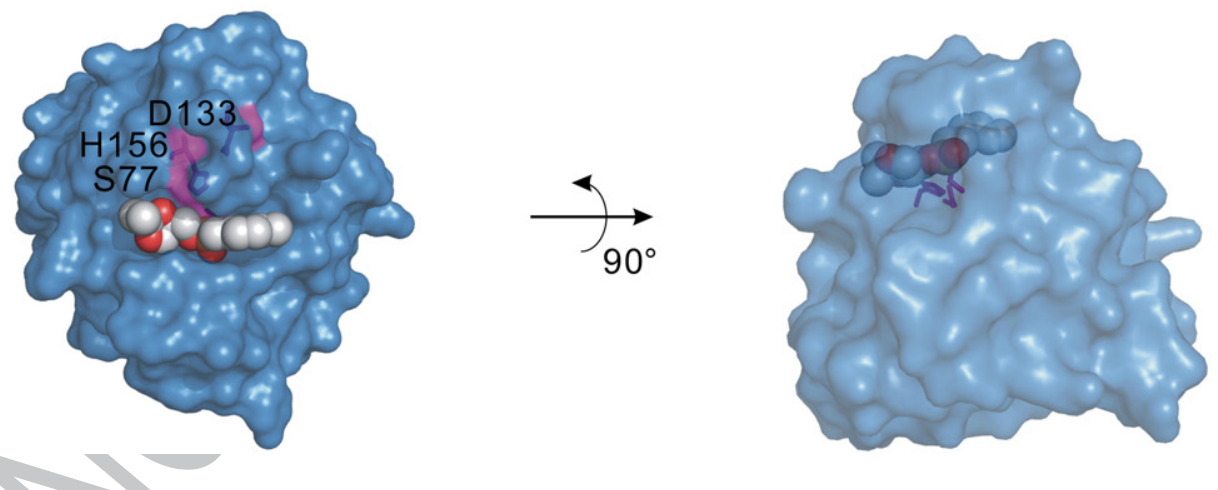

Fig. 4. The substrate binding site of AFL and BSL. (a) The top view and the side view of AFL. Left: the top view of the AFL structure is shown as a surface with the $\mathrm{N}$-terminal domain in blue, the $\mathrm{C}$-terminal domain in green, the lid domain in orange, and the substrate covering motif in cyan. Right: the side view of the AFL structure is obtained by rotating the top view by $90^{\circ}$, with a horizontal axis in the plane of paper. The substrate is shown as a gray sphere. (b) The top view of AFL without the lid domain. The top view of AFL structure is colored as in (a). The catalytic triad is shown as a purple line. (c) The top view of AFL without the lid domain and the substrate covering motif. The top view of the AFL structure is shown in a surface colored as in (a). The catalytic triad is shown as a purple stick. (d) The top view and the side view of BSL. Left: the BSL structure is shown as the surface in blue with its substrate shown as gray spheres and the triad shown as a purple stick. Right: the side view of BSL is obtained by rotating the top view by $90^{\circ}$, with a horizontal axis in the plane of paper.

between the substrate and the outer environment. Access of the substrate to the binding pocket is only possible through an opening of the lid on top of the $\mathrm{N}$-terminal domain. The four helices in the lid might undergo conformational change due to the contact with substrate to allow the entrance of substrate 358 (Fig. 4b). In the AFL lipase, we observed that the 359 carbonyl group of the ligand is facing the catalytic 360 triad and the acyl part extends to the C-terminal 361 domain (Fig. 4c). We also observed some space at the 362 
end of the tunnel toward the C-terminal domain occupied by some water molecule. This shows that the tunnel might be solvent accessible. Whether some conformational change of the substrate covering motif is necessary for the substrate to enter the binding pocket awaits further investigation.

To understand the binding of a lipid substrate in AFL, we compared the structure of AFL with the bound fatty acid fragment with the BSL structure 371 complexed with the covalently bound inhibitor Rc- 372 IPG phosphonate inhibitor. ${ }^{22}$ The acyl part binding 373 pocket in AFL is much larger when compared with 374 that of BSL, and consequently, the enzyme-substrate 375 interactions are more extensive than those in BSL. In 376 addition, the ligand binding direction in the active 377 site of AFL and BSL is opposite to each other. In other 378 (a)

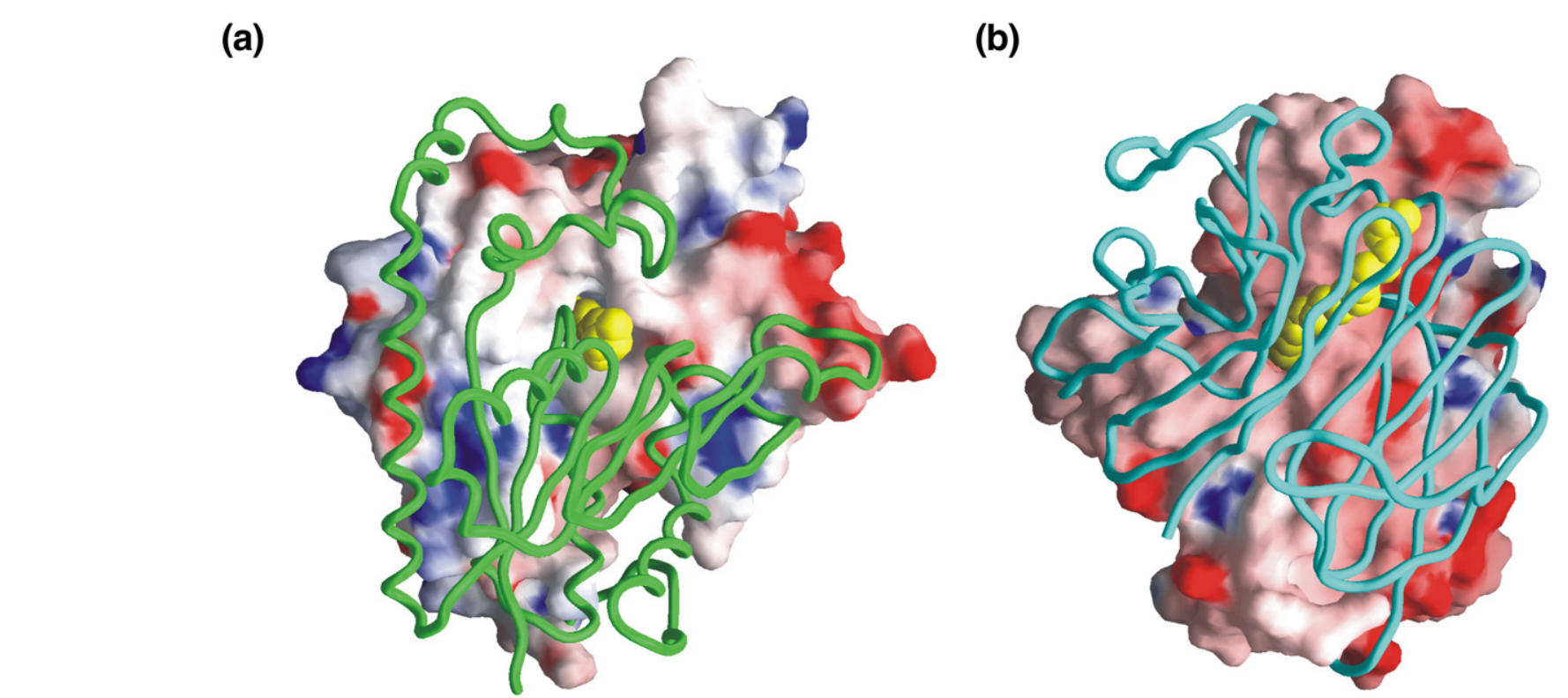

(c)

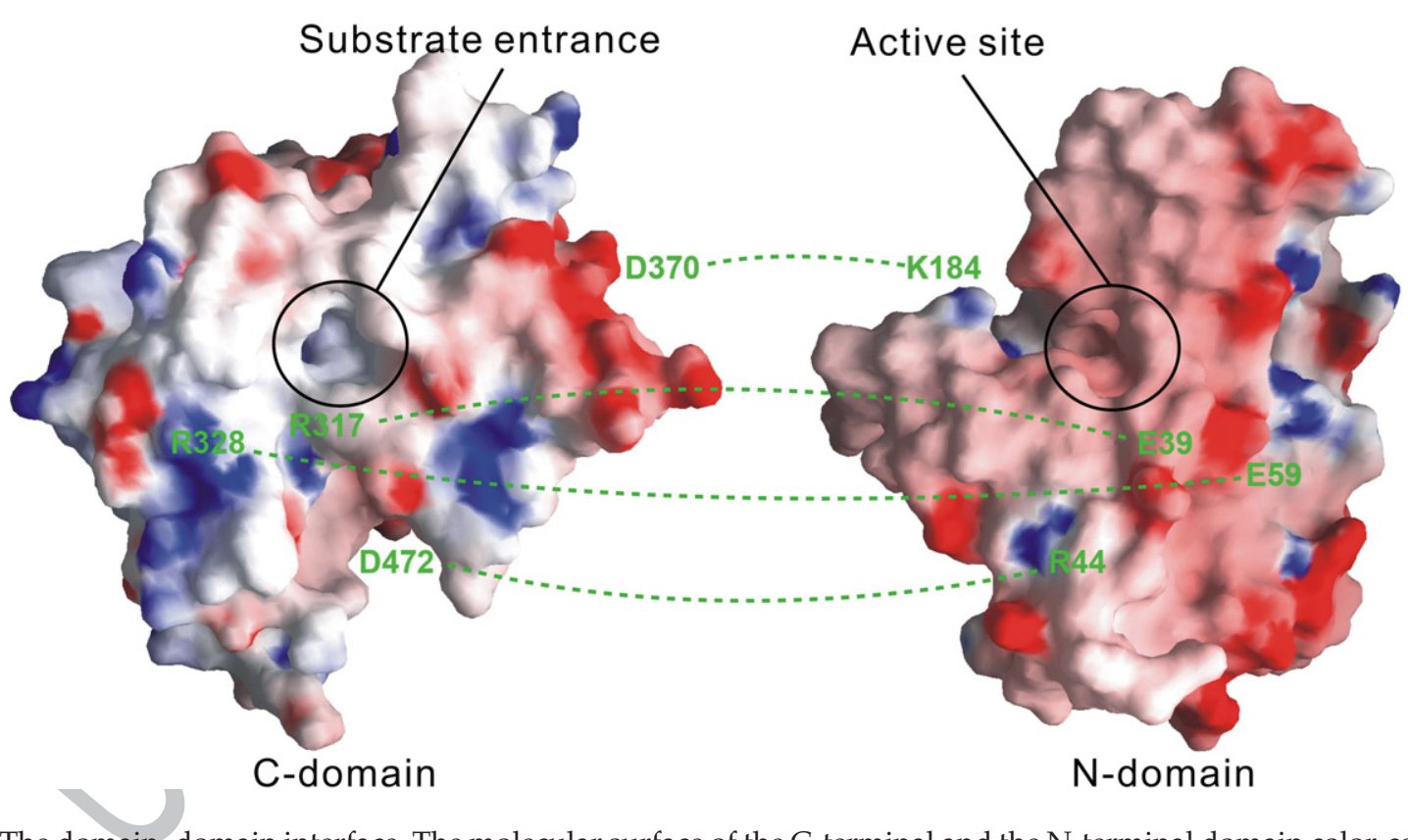

(b)

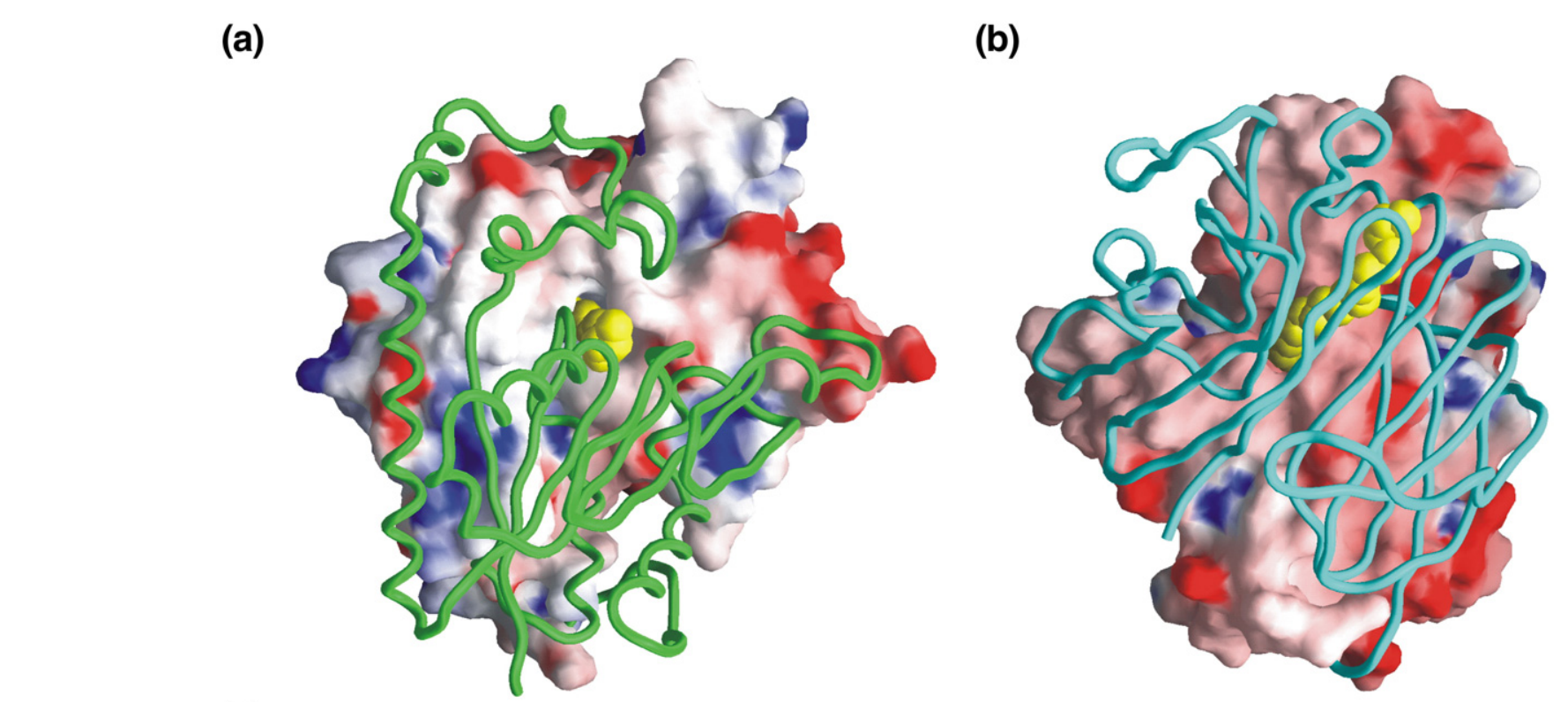

\section{Active site}

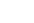


words, the acyl part of BSL ligand extends to the catalytic N-terminal domain, which is in a completely opposite direction to that of AFL (Fig. 4d).

\section{The domain-domain interface}

The residues involved in the interactions can be identified by calculation of the solvent-accessible surface areas before and after domain-domain interaction. The exposed and buried surfaces were calculated with Areaimol in CCP4 using a solvent probe radius of $1.4 \AA^{23}$ The solvent-accessible surface areas calculated for each of the domains are $2049 \AA^{2}$ or $17 \%$ for the C-terminal domain and $2031 \AA^{2}$ or $18 \%$ for the N-terminal domain of AFL (Supplementary Table 2). Figure 5 shows the molecular surface at the interface of the two domains with color coded by electrostatic potential. The interacting surfaces are highly hydrophobic and spotted with charged areas (Fig. 5a and b). Interestingly, the charges are complementary from the two domains. There are four ion pairs (Glu39-Arg317, Arg44-Asp472, Glu59-Arg328, and Lys184-Asp370) involved in the surface interactions (Fig. 5c). The detailed interactions of the four ion pairs are shown in Supplementary Fig. 2. The electrostatic interactions must play one of the most important roles in the domain-domain interactions with which the tight bipartite structure is formed, resulting in a highly thermostable structure. In addition to the complementary charge pair between the two domains, extensive hydrophobic interaction also plays an important role in stabilizing the structure of AFL. There are 12 and 21 amino acids from the Nand C-terminal domains, respectively, participating in the hydrophobic interaction (Supplementary Table 2). Thus, our analysis suggests that the higher thermostability of mature AFL (mAFL) as compared to C-domain-truncated AFL (tAFL) may be attributed to a larger buried surface area and extra electrostatic networks embedded in the nonpolar interface between the $\mathrm{C}$-terminal and the $\mathrm{N}$-terminal domains in the mAFL enzyme.

\section{AFL is a true lipase that contains a unique C- domain structure for substrate binding and catalysis}

As shown in Fig. 6a, interfacial activation effect of AFL was observed with tricaprylin as a substrate. A drastic increase in lipase activity occurred when the solubility limit of tricaprylin was exceeded. This means that the apparent rate of hydrolysis correlates with the degree of micellar formation. Consistent with observations from other lipases, the lid conformation may change from the closed to the open form in the presence of lipid interface. Together with the evidence of the lid structure in AFL, we classify AFL as a lipase rather than as a carboxylesterase.

To obtain insight into the function of the Cterminal domain and the substrate preference of AFL, we performed activity measurements using

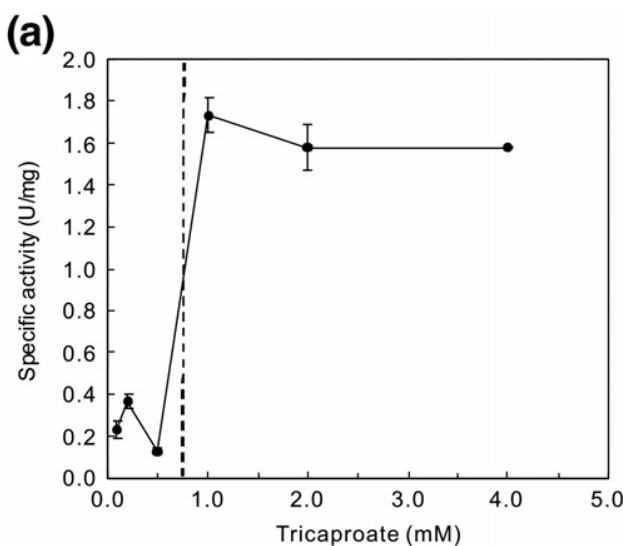

(b)

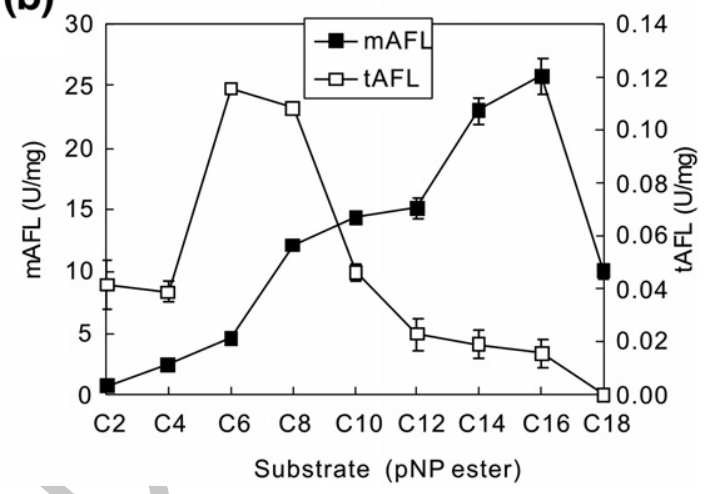

Fig. 6. (a) Interfacial activation effect of AFL. The enzyme was assayed in the presence of different tricaprylin concentrations under the conditions of lipase activity assay. The broken line indicates the limit of solubility of tricaprylin. (b) Substrate specificity of recombinant AFL $(\mathrm{mAFL}, \square)$ and tAFL $(\square)$ in the hydrolysis of $p$-nitrophenyl $(\mathrm{pNP})$ esters containing fatty acids of various chain lengths. One unit of esterase activity is the amount of enzyme that hydrolyzes $1.0 \mu \mathrm{mol}$ of $\mathrm{pNP}$ ester per minute at $60{ }^{\circ} \mathrm{C}$ and $\mathrm{pH}$ 8.0. Values are means $\pm \mathrm{SD}$ from three independent experiments.

pNP ester as the substrate to compare the substrate 438 spectrum between the mAFL (no signal peptide) 439 and tAFL. Activity measurements are provided in 440 Fig. 6b. The mAFL preferentially hydrolyzed esters 441 of long-chain fatty acids (C10-C16) and especially 442 those of C16. In contrast, tAFL preferentially 443 hydrolyzed esters of short-chain fatty acids (C4- 444 C10) and especially those of C6, but with a much 445 lower activity. Without the C-terminal domain, AFL 446 lost its function to hydrolyze long-chain ester 447 substrates. Although mAFL shows its highest 448 activity at a substrate length of C16, it still retains 449 a lower activity to substrate with longer chain length 450 such as C18. These results suggest that the C- 451 terminal domain of AFL is essential for its binding to 452 the long-chain substrate.

To further explore the function of the unique C- 454 terminal domain, we also investigated its role in 455 thermostability and $\mathrm{pH}$ stability. We discovered that 456 the deletion of the C-terminal domain reduces the 457 optimal temperature from 90 to $80^{\circ} \mathrm{C}$ (Fig. 7a). The 458 lack of the C-terminal domain also causes the 459 
(a)

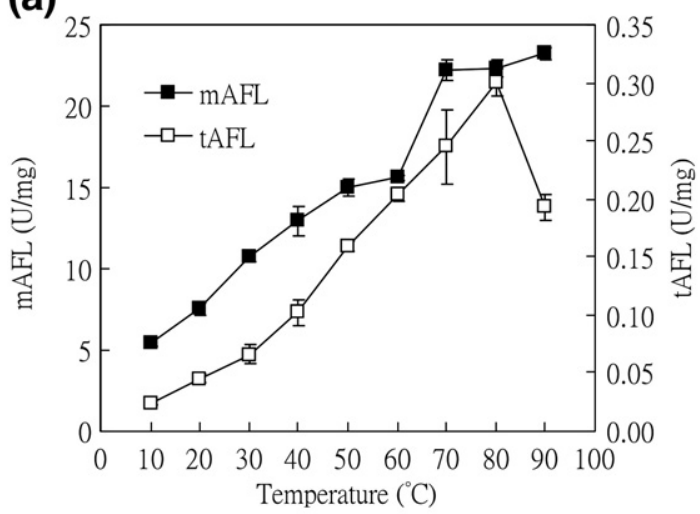

(c)

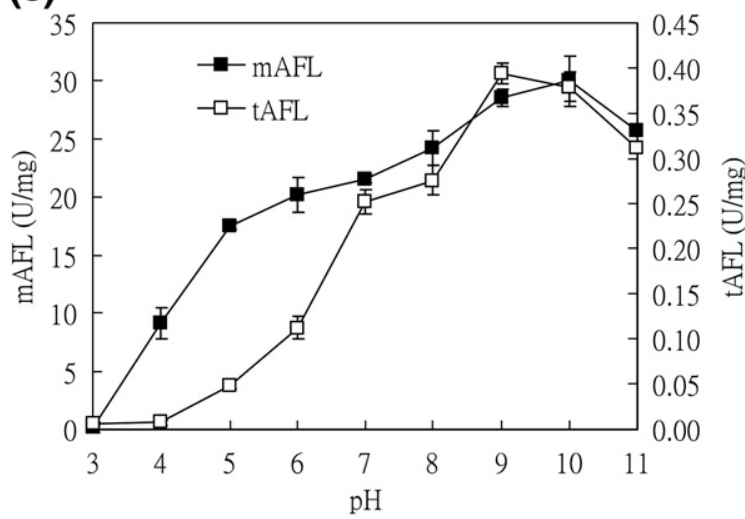

(b)

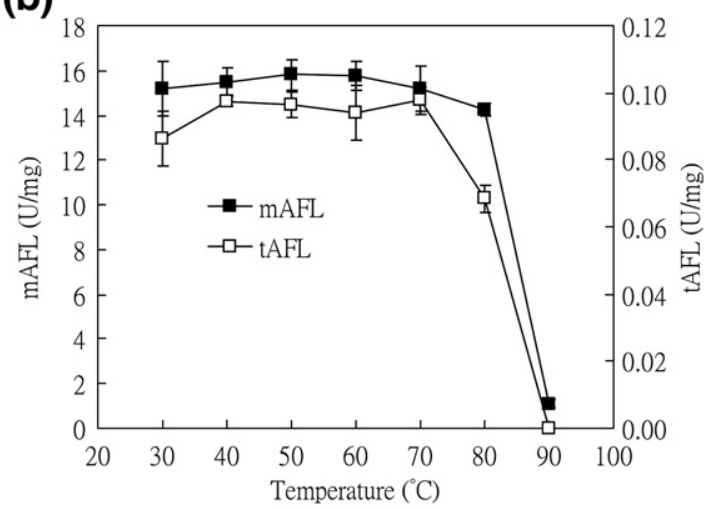

(d)

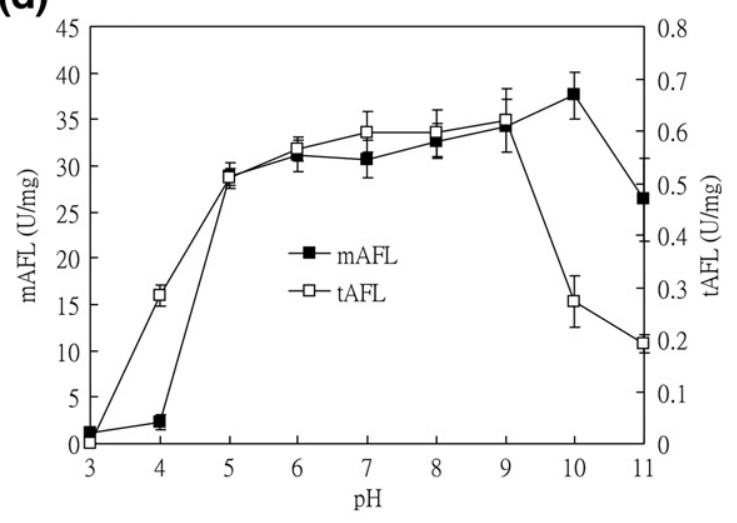

Fig. 7. Optimum temperature, thermostability, optimum $\mathrm{pH}$, and $\mathrm{pH}$-stability assay of recombinant AFL (mAFL, and tAFL ( $\square$ ). (a) Determination of optimum temperature. The optimal temperature was investigated in the range of 10$90^{\circ} \mathrm{C}$ at $\mathrm{pH} 8.5$ using $p$-nitrophenyl caprate $(0.2 \mathrm{mM})$ as a substrate. Optimal temperature: $90^{\circ} \mathrm{C}, 23.27 \mathrm{U} / \mathrm{mg}$ for $\mathrm{mAFL}$; $80^{\circ} \mathrm{C}, 0.30 \mathrm{U} / \mathrm{mg}$ for tAFL. (b) Thermostability assay. To analyze the thermostability of AFL, we incubated the lipase enzyme for $30 \mathrm{~min}$ at various temperatures in the range of $30-90{ }^{\circ} \mathrm{C}$. Residual activity was determined at $60{ }^{\circ} \mathrm{C}, \mathrm{pH} 8.5$, using $\mathrm{pNPP}(0.2 \mathrm{mM})$ as a substrate. (c) Determination of optimum $\mathrm{pH}$. The optimal $\mathrm{pH}$ was investigated in the $\mathrm{pH}$ range of 3.0-11.0 at $60{ }^{\circ} \mathrm{C}$ using Good's buffer [ $50 \mathrm{mM}$ each of $N, N$-bis(2-hydroxyethyl)glycine, CAPS, sodium acetate, and BisTris propane] containing $0.5 \%$ Triton X-100. $\mathrm{pH}$ effects were assessed using $\mathrm{pNPP}(0.2 \mathrm{mM})$ as a substrate for $\mathrm{mAFL}$ and $p$ nitrophenyl caproate $(0.5 \mathrm{mM})$ for tAFL. Optimal $\mathrm{pH}$ : $\mathrm{pH} 10.0,30.02 \mathrm{U} / \mathrm{mg}$ for mAFL; $\mathrm{pH} 9.0,0.39 \mathrm{U} / \mathrm{mg}$ for tAFL. (d) $\mathrm{pH}$-stability assay. The $\mathrm{pH}$ stability of AFL was determined by incubating the enzyme preparation in buffers of different $\mathrm{pH}$ values in the range of 3.0-11.0 for $2 \mathrm{~h}$ at $60^{\circ} \mathrm{C}$. Residual activity was determined at $\mathrm{pH} 8.5$ at $60^{\circ} \mathrm{C}$ using pNPP $(0.2 \mathrm{mM})$ as a substrate for $\mathrm{mAFL}$ and $p$-nitrophenyl caproate $(0.5 \mathrm{mM})$ for tAFL.

protein to be less thermostable (the activity drastically reduced above $70^{\circ} \mathrm{C}$ in tAFL instead of $80^{\circ} \mathrm{C}$ in mAFL) (Fig. 7b). However, the deletion also changes the optimal $\mathrm{pH}$ and $\mathrm{pH}$ stability of mAFL. It reduces the optimal pH from 10.0 to 9.0 (Fig. 7c) and causes the protein to be less $\mathrm{pH}$ stable (the activity drastically reduced above $\mathrm{pH} 10.0$ in tAFL instead of $\mathrm{pH} 11.0$ in mAFL) (Fig. 7d). Compared to the Cterminal domain's role in thermostability and $\mathrm{pH}$ stability, it appears that this domain plays a more important role in catalytic efficiency. Under the optimal temperature and optimal $\mathrm{pH}$, the activity of $\mathrm{mAFL}$ is around 77 times higher than that of tAFL. Moreover, the deletion also slightly changes the optimal temperature and $\mathrm{pH}$ of mAFL. Therefore, it is likely that the C-terminal domain not only plays an important role in substrate specificity and catalytic efficiency but also attributes partly to its thermophilicity and alkalophilicity.

In an earlier article, ${ }^{14}$ the authors used an inactive premature form of AFL ( $\mathrm{pAFL}$ ), which contains a signal peptide and shows low activity towards longchain triglycerides. We have measured the specific activities (in units per milligram) of mAFL and pAFL using the colorimetric pNPP assay. mAFL shows a 34-fold higher activity than pAFL at $60^{\circ} \mathrm{C}$. As shown in Fig. 7a, mAFL shows high activities in the temperature range from 70 to $90^{\circ} \mathrm{C}$, while pAFL has the highest activity at $70{ }^{\circ} \mathrm{C}$ in the article by Rusnak et al. ${ }^{14}$ In Fig. $7 \mathrm{~b}, \mathrm{mAFL}$ has high residual activity after incubation at the temperatures up to $80{ }^{\circ} \mathrm{C}$ (at least $90 \%$ ), while pAFL shows low residual activity even at $40{ }^{\circ} \mathrm{C}$ (about $40 \%$ ). Obviously, these two forms of AFL display different optimal temperatures and thermostabilities.

The presence of a C-terminal non-catalytic domain of about 200 residues for substrate binding distinguishes AFL from all other lipases whose structures have been determined. The AFL is the first lipase structure discovered so far to contain a C-terminal domain, which enables the binding of long-chain substrate. Although there are several lipases with a 
C-terminal domain, their C-domain function and composition are totally different from that of AFL. For example, pancreatic lipases (PLs) contain a Cterminal domain belonging to the $\mathrm{C} 2$ family, which is a $\beta$-sandwich composed of around 100 amino acids. The $\beta$-sandwich C-terminal domain of PL plays an important part in the binding process between the PL and colipase, the specific PL cofactor. ${ }^{24}$ The C-terminal domains of AFL and PL not only differ in their function but also show a large difference in their size and the interaction with the $\mathrm{N}$-terminal domain. The C-terminal domain of AFL forms extensive interaction with the $\mathrm{N}$-terminal domain to enable the binding of long-chain substrate and help protein stability. However, the Cdomain of PL does not interact extensively with the $\mathrm{N}$-domain; neither does it participate in substrate binding. A search of the C-terminal domain of AFL with DALI uncovered a number of similar structures in the Protein Data Bank (PDB) with Z scores ranging from 7.0 to 2.0. Among these structures, many have not been functionally characterized and, hence, did not provide any useful functional information. However, 5 out of the top 10 hits with known functions are transthyretins (TTRs). ${ }^{25-29}$ TTR is a tetrameric protein composed of four identical subunits, each consisting of 8 antiparallel $\beta$-strands, in contrast to the C-terminal domain of AFL with 14 $\beta$-strands. The substrate binding modes of AFL and TTR also show a large difference. The function of TTR is to transport thyroid hormones and other hydrophobic substances in the blood. Although the C-terminal domain of AFL does not superimpose well with the TTR proteins, their similarity in folding pattern infers that the C-terminal domain of AFL might be able to bind or transport hydrophobic substances.

In conclusion, the unique C-terminal domain in AFL enables it to bind and react with long-chain substrates that probably form in the growing environment of $A$. fulgidus. This unique domain also helps to increase AFL's catalytic efficiency to a large extent. Furthermore, the presence of the extensive inter-domain interactions contributes to the thermostability of AFL. As deduced from the biochemical data and crystal structures, we defined the role of the C-terminal $\beta$-barrel domain of the AFL as an anchoring domain for its substrate as well as providing the stability force. The observation of the $\mathrm{pH}$-dependent conformational changes associated with the two hinge residues connecting the lid domain and the catalytic domain may explain the alkalophilic nature of this novel lipase.

\section{Materials and Methods}

\section{Materials}

PfuTurbo DNA polymerase was obtained from Invitrogen (Carlsbad, CA). The plasmid miniprep kit, DNA gel extraction kit, and $\mathrm{Ni}^{2+}$-NTA resin were purchased from Qiagen (Valencia, CA). The protein expression kit [includ- ing the pET23a vector and competent JM109 and BL21 561 (DE3) cells] was obtained from Novagen (Madison, WI). 562 The QuikChange Site-Directed Mutagenesis Kit was 563 obtained from Stratagene, Inc. (La Jolla, CA). All commer- 564 cial buffers and reagents were of the highest grade.

Protein expression and purification of the truncated, native, and mutant AFL

AFL gene was amplified by PCR from genomic DNA of 568 A. fulgidus with primers 5'-AFL (5'-ATACATATGGCG- 569 GAAGACTTTAGACCG-3') and $3^{\prime}$-AFL (5'-CAGTCGA- 570 CATTAAATGTAATCCGAAAA-3') (restriction sites are 571 underlined). The PCR product encoding AFL was cloned 572 into expression vector pET-23a (Novagen), and DNA 573 sequencing was performed to confirm the appropriate 574 orientation. This construct, with a (His) 6 tag attached to 575 the C-terminal, was transferred into E. coli BL21 (DE3) 576 (Novagen) competent cells.

The hexa-His-tagged wild-type and mutant AFL proteins were overexpressed in BL21 (DE3). Cells were cultured in Difco Luria-Bertani broth containing $50 \mathrm{mg} / \mathrm{L}$ ampicillin to an optical density at $600 \mathrm{~nm}$ of $0.5-0.6$ and then induced with $0.5 \mathrm{mM}$ isopropyl- $\beta$-D-thiogalactopyranoside to induce protein expression. Cells were grown for $15 \mathrm{~h}$ at $30{ }^{\circ} \mathrm{C}$. The cells were then harvested by centrifugation at $4000 \mathrm{~g}$ for $15 \mathrm{~min}$ and disrupted by a French press in a buffer containing $25 \mathrm{mM}$ Tris- $\mathrm{HCl}$ $(\mathrm{pH} 7.5), 150 \mathrm{mM} \mathrm{NaCl}$, and $20 \mathrm{mM}$ imidazole. The homogenate was centrifuged at $27,000 \mathrm{~g}$ for $30 \mathrm{~min}$, and the supernatant was recovered as a crude extract, which was then heated at $60{ }^{\circ} \mathrm{C}$ for $30 \mathrm{~min}$ to denature unwanted proteins. The denatured proteins were removed by centrifugation at $27,000 \mathrm{~g}$ for $30 \mathrm{~min}$. The supernatant containing the thermo-tolerant AFL was recovered, and the cell-free extract was loaded onto a $\mathrm{Ni}^{2+}$-NTA column, which had been equilibrated with lysis buffer. The column was washed with lysis buffer, and the (His) ${ }_{6}$-tagged AFL was subsequently eluted by a linear gradient of imidazole concentration from 30 to $500 \mathrm{mM}$. The enzyme was dialyzed 3 times each with $5 \mathrm{~L}$ of buffer $(20 \mathrm{mM}$ Tris- $\mathrm{HCl}$, $\mathrm{pH}$ 8.0) for $6 \mathrm{~h}$. The resulting AFL solution was further purified by applying it to a diethylaminoethyl cellulose column $(2.2 \mathrm{~cm} \times 50 \mathrm{~cm})$ equilibrated with $20 \mathrm{mM}$ Tris$\mathrm{HCl}(\mathrm{pH}$ 8.0). The column was washed with $500 \mathrm{~mL}$ of the same buffer containing $50 \mathrm{mM} \mathrm{NaCl}$, and AFL proteins were then eluted with $1000 \mathrm{~mL}$ of a linear gradient of 50 to $500 \mathrm{mM} \mathrm{NaCl}$ in the same buffer. The purified (His) tagged AFL was dialyzed three times against $5 \mathrm{~L}$ of buffer (20 mM Tris- $\mathrm{HCl}, \mathrm{pH} 8.0$ ), concentrated by Centricon Plus-20 with 10 kDa cutoff membrane (Millipore, Massachusetts, USA), and stored at $-80^{\circ} \mathrm{C}$. The purities $(>95 \%)$ were estimated by SDS/PAGE.

\section{Crystallization and data collection for AFL}

AFL crystals were obtained in three different conditions 613 using the hanging drop method (Hampton Research, 614 Laguna Niguel, CA). Native (isopropanol) and S136A AFL 615 crystals were obtained by mixing $2 \mu \mathrm{L}$ of an AFL solution 616 (5-10 mg/mL AFL in $20 \mathrm{mM}$ Tris-HCl, $\mathrm{pH}$ 8.0) with $2 \mu \mathrm{L} \quad 617$ of mother liquor $\left(0.2 \mathrm{M} \mathrm{CaCl}_{2}, 0.1 \mathrm{M}\right.$ sodium acetate, 618 $\mathrm{pH}$ 4.6, and $10 \%$ isopropanol), and equilibrating with 619 $500 \mu \mathrm{L}$ of the mother liquor. Native (PEG 4000) AFL 620 crystal was crystallized by mixing $2 \mu \mathrm{L}$ of an AFL solution 621 (5-10 mg/mL AFL in $20 \mathrm{mM}$ Hepes, $\mathrm{pH}$ 7.5) with $2 \mu \mathrm{L}$ of 622 mother liquor $\left(0.16 \mathrm{M} \mathrm{MgCl}_{2}, 0.08 \mathrm{M}\right.$ Tris- $\mathrm{HCl}, \mathrm{pH} 8.5,623$ $24 \%$ PEG 4000, and 20\% glycerol). The native crystals 624 
(PEG 8000) were obtained by mixing $2 \mu \mathrm{L}$ of an AFL solution (5-10 mg/mL AFL in $20 \mathrm{mM}$ Hepes, $\mathrm{pH} 7.5$ ) with $2 \mu \mathrm{L}$ of mother liquor (20\% PEG 8000, $100 \mathrm{mM}$ CAPS, $\mathrm{pH} 10.5$, and $200 \mathrm{mM} \mathrm{NaCl}$ ). The Form I crystals grew to $0.5 \mathrm{~mm} \times 0.2 \mathrm{~mm} \times 0.2 \mathrm{~mm}$ in 4 days, at room temperature, and then were soaked briefly in solution containing crystallization buffer with $10 \%(\mathrm{v} / \mathrm{v})$ perfluoropolyether as cryoprotectant.

X-ray diffraction data were collected at beam line BL13B1 of the National Synchrotron Radiation Research Center (NSRRC, Hsinchu, Taiwan), Taiwan Contract BL12B2 station at SPring-8 (Hyogo, Japan), and Advanced Light Source (Lawrence Berkeley National Laboratory). Diffraction data were processed and scaled by using the program HKL2000. ${ }^{30}$ The data sets we obtained can be divided into three different crystal forms. The native AFL crystal (isopropanol), the S136A mutant crystal, the AFL$\mathrm{KAu}(\mathrm{CN})_{2}$, and the AFL- $\mathrm{K}_{2} \mathrm{PtCl}_{4}$ crystals belonged to the $P 2{ }_{1} 22_{1}$ space group with typical unit cell parameters of $a=89-91 \AA, b=105-107 \AA$, and $c=117-118 \AA$ (Form I). Each asymmetric unit contained two AFL molecules. The native (PEG 4000) crystal belonged to the $P 2_{1} 2_{1} 2_{1}$ space group with unit cell parameters of $a=52 \AA, b=106 \AA$, and $c=175 \AA$ (Form II). There are also two AFL molecules in each asymmetric unit. The other native AFL crystal (PEG 8000 ) in the $\mathrm{P}_{3}$ space group had typical unit cell parameters of $a=b=99.55 \AA$ and $c=59.02 \AA$ (Form III). Each asymmetric unit contained one AFL molecule.

\section{Structure determination and refinement}

Two AFL MIR data sets were collected at beam line BL13B1 of the NSRRC. The phase angles of the AFL crystal were calculated using $\mathrm{KAu}(\mathrm{CN})_{2}$ and $\mathrm{K}_{2} \mathrm{PtCl}_{4}$ heavy-atom derivative data by the MIR method using SOLVE ${ }^{31}$ (Supplementary Table 1). The MIR map at $1.83 \AA$ was subject to maximum likelihood density modification followed by autotracing using RESOLVE. ${ }^{32}$ An initial model was built using $\mathrm{O}^{33}$ Density modification with non-crystallographic symmetry averaging, using the program CNS, ${ }^{34}$ improved the phase angles and enabled construction of a more complete model by RESOLVE. Manual building of the remaining model and further refinement were carried out with the programs $\mathrm{O}$, XtalView, ${ }^{35}$ and CNS. Prior to use in structural refinements, $5 \%$ of the randomly selected reflections were set aside for calculating $R_{\text {free }}$ as a quality monitor. ${ }^{36}$ The other three crystal structures were determined by using molecular replacement. Refinement statistics of AFL are summarized in Table 1. The residues in the generously allowed and disallowed region are $0.5 \%$. This is because residues Ser136/Ala136 and Ala255 form glycine-like conformation in all four AFL structures. The glycine-like conformation plays an important role in the function of the active site. The molecular figures were produced by using PyMOL (DeLano Scientific) $\$$.

\section{Site-directed mutagenesis of AFL}

AFL mutant S136A was prepared by using a QuikChange Site-Directed Mutagenesis Kit in conjunction with the AFL gene template in the pET-23a (+) vector. The basic procedure of mutagenesis utilizes a supercoiled doublestranded DNA vector with an insert of interest and two synthetic oligonucleotide primers containing the desired

\$http:/ / www.pymol.org mutation. The mutation was confirmed by sequencing the 686 entire AFL mutant gene of the plasmid obtained from the 687 overnight culture. The correct construct was subsequently 688 transformed to E. coli BL21 (DE3) for protein expression 689 and purification.

\section{Construction of the C-terminal-truncated mutant}

To truncate the AFL open reading frame by engineering 692 a terminator after amino acid T235, we PCR amplified a 693 DNA fragment from the full-length AFL using a sense 694 primer (5'-ATACATATGGCGGAAGACTTTAGACCG- 695 $3^{\prime}$ ) and an antisense primer (5'-CAGTCGACAT- 696 TAGGTTGTTGCTGGCTTGTA-3') (restriction sites are 697 underlined). The sequence of the antisense primer was 698 complementary to nucleotides 688 to 705 of the AFL open 699 reading frame and included one engineered stop codon. 700 An NdeI-SalI-treated DNA fragment of PCR product was 701 ligated into the pET-23a (+) vector.

\section{Enzyme assay}

The initial rates of lipase activities were measured spec- 704 trophotometrically (Hitachi U-2010) using p-nitrophenyl 705 esters as substrates. The hydrolysis of $p$-nitrophenyl esters 706 was carried out at $60^{\circ} \mathrm{C}$ in $500 \mu \mathrm{L}$ of reaction buffer $(20 \mathrm{mM} \quad 707$ Tris $-\mathrm{HCl}, 0.1 \%$ gum arabic, and $0.2 \%$ sodium deoxycho- 708 late, $\mathrm{pH} 8.5)$ containing the correspondent $p$-nitrophenyl 709 ester $(0.2 \mathrm{mM})$. The increase in absorbance was recorded 710 for $10 \mathrm{~min}$ at $405 \mathrm{~nm}$. One unit of activity was defined as 711 the quantity of enzyme necessary to release $1 \mu \mathrm{mol}$ of 712 $p$-nitrophenol per minute under the above conditions. 713

\section{Interfacial activation assay}

The lipolytic activity was evaluated titrimetrically using 715 tricaprylin $\left(C_{8: 0}\right)$ as a substrate. The release of free fatty 716 acids was monitored continuously by titration using 717 $10 \mathrm{mM} \mathrm{NaOH}$ with a pH-Stat (Radiometer TIM854, 718 Copenhagen, Bagsvaerd, Denmark), adjusted to a con- 719 stant end-point value of $\mathrm{pH}$ 8.0. The substrate emulsifica- 720 tion reagent contains $60 \mathrm{mM} \mathrm{NaCl}, 0.4 \mathrm{mM}$ Tris, $10.8 \% \quad 721$ glycerol, and $0.4 \%$ gum arabic. Each assay was carried out 722 in a $60{ }^{\circ} \mathrm{C}$ thermostatted reaction vessel containing $20 \mathrm{~mL} \quad 723$ of various concentrations of substrate emulsion. One unit 724 of lipase activity was defined as the amount of lipase 725 necessary to produce $1 \mu \mathrm{mol}$ of fatty acid per minute 726 under the assay conditions.

\section{PDB accession numbers}

The coordinates of native AFL (isopropanol), native 729 AFL (PEG 4000), native AFL (PEG 8000), and mutant AFL 730 S136A (isopropanol) have been deposited in the PDB and 731 are available under accession numbers 2ZYI, 2ZYR, 2ZYS, 732 and $2 \mathrm{ZYH}$, respectively.

\section{Acknowledgements}

We thank NSRRC (Taiwan), Advanced Light 735 Source (USA), and SPring-8 (Japan) for beam time 736 allocation and Hui-Ling Shr at the National Core 737 
Facility of X-ray Crystallography, the Institute of Biological Chemistry, Academia Sinica, for crystal screening.

\section{Supplementary Data}

Supplementary data associated with this article can be found, in the online version, at doi:10.1016/ j.jmb.2009.05.017

\section{References}

1. Schrag, J. D. \& Cygler, M. (1997). Lipases and alpha/ beta hydrolase fold. Methods Enzymol. 284, 85-107.

2. Jaeger, K. E., Ransac, S., Dijkstra, B. W., Colson, C., van Heuvel, M. \& Misset, O. (1994). Bacterial lipases. FEMS Microbiol. Rev. 15, 29-63.

3. Bornscheuer, U. T., Altenbuchner, J. \& Meyer, H. H. (1999). Directed evolution of an esterase: screening of enzyme libraries based on $\mathrm{pH}$-indicators and a growth assay. Bioorg. Med. Chem. 7, 2169-2173.

4. Houde, A., Kademi, A. \& Leblanc, D. (2004). Lipases and their industrial applications: an overview. Appl. Biochem. Biotechnol. 118, 155-170.

5. Jaeger, K. E. \& Reetz, M. T. (1998). Microbial lipases form versatile tools for biotechnology. Trends Biotechnol. 16, 396-403.

6. Nardini, M. \& Dijkstra, B. W. (1999). Alpha/beta hydrolase fold enzymes: the family keeps growing. Curr. Opin. Struct. Biol. 9, 732-737.

7. Arpigny, J. L. \& Jaeger, K. E. (1999). Bacterial lipolytic enzymes: classification and properties. Biochem. J.343, 177-183.

8. Gilbert, E. J. (1993). Pseudomonas lipases: biochemical properties and molecular cloning. Enzyme Microb Technol. 15, 634-645.

9. Svendsen, A., Borch, K., Barfoed, M., Nielsen, T. B., Gormsen, E. \& Patkar, S. A. (1995). Biochemical properties of cloned lipases from the Pseudomonas family. Biochim. Biophys. Acta, 1259, 9-17.

10. Gupta, R., Gupta, N. \& Rathi, P. (2004). Bacterial lipases: an overview of production, purification and biochemical properties. Appl. Microbiol. Biotechnol. 64, 763-781.

11. Beeder, J., Nilsen, R. K., Rosnes, J. T., Torsvik, T. \& Lien, T. (1994). Archaeoglobus fulgidus isolated from hot North Sea oil field waters. Appl. Environ. Microbiol. 60, 1227-1231.

12. Turner, P., Mamo, G. \& Karlsson, E. N. (2007). Potential and utilization of thermophiles and thermostable enzymes in biorefining. Microb. Cell Fact. 6, 9-23.

13. Yano, J. K. \& Poulos, T. L. (2003). New understandings of thermostable and peizostable enzymes. Curr. Opin. Biotechnol. 14, 360-365.

14. Rusnak, M., Nieveler, J., Schmid, R. D. \& Petri, R. (2005). The putative lipase, AF1763, from Archaeoglobus fulgidus is a carboxylesterase with a very high $\mathrm{pH}$ optimum. Biotechnol. Lett. 27, 743-748.

15. Dartois, V., Coppée, J. Y., Colson, C. \& Baulard, A. (1994). Genetic analysis and overexpression of lipolytic activity in Bacillus subtilis. Appl. Environ. Microbiol. 60, 1670-1673.

16. Jaeger, K. E., Dijkstra, B. W. \& Reetz, M. T. (1999). Bacterial biocatalysts: molecular biology, three- dimensional structures, and biotechnological applica- 799 tions of lipases. Annu. Rev. Microbiol. 53, 315-351. 800

17. Gotz, F., Verheij, H. M. \& Rosenstein, R. (1998). 801 Staphylococcal lipases: molecular characterisation, 802 secretion, and processing. Chem. Phys. Lipids, 93, 15-25. 803

18. Rosenstein, R. \& Gotz, F. (2000). Staphylococcal 804 lipases: biochemical and molecular characterization. 805 Biochimie, 82, 1005-1014.

19. Gupta, R., Gupta, N. \& Rathi, P. (2004). Bacterial 807 lipases: an overview of production, purification and 808 biochemical properties. Appl. Microbiol. Biotechnol. 64, 809 763-781.

20. Guex, N. \& Peitsch, M. C. (1997). SWISS-MODEL and 811 the Swiss-PdbViewer: an environment for compara- 812 tive protein modeling. Electrophoresis, 18, 2714-2723. 813

21. Ollis, D. L., Cheah, E., Cygler, M., Dijkstra, B., Frolow, 814 F., Franken, S. M. et al. (1992). The alpha/beta 815 hydrolase fold. Protein Eng. 5, 197-211. 816

22. Droge, M. J., Boersma, Y. L., van Pouderoyen, G., 817 Vrenken, T. E., Ruggeberg, C. J., Reetz, M. T. et al. 818 (2006). Directed evolution of Bacillus subtilis lipase A 819 by use of enantiomeric phosphonate inhibitors: crystal 820 structures and phage display selection. ChemBioChem, 821 7, 149-157.

23. Collaborative Computational Project Number 4. 823 (1994). The CCP4 suite: programs for protein crystal- 824 lography. Acta Crystallogr., Sect. D: Biol. Crystallogr. 50, 825 760-763.

24. Chahinian, H., Sias, B. \& Carriere, F. (2000). The C- 827 terminal domain of pancreatic lipase: functional and 828 structural analogies with c2 domains. Curr. Protein 829 Pept. Sci. 1, 91-103.

25. Zanotti, G., Cendron, L., Ramazzina, I., Folli, C., 831 Percudani, R. \& Berni, R. (2006). Structure of zebra fish 832 HIUase: insights into evolution of an enzyme to a 833 hormone transporter. J. Mol. Biol. 363, 1-9. 834

26. Muziol, T., Cody, V. \& Wojtczak, A. (2001). Compar- 835 ison of binding interactions of dibromoflavonoids 836 with transthyretin. Acta Biochim. Pol. 48, 885-892. 837

27. Jung, D. K., Lee, Y., Park, S. G., Park, B. C., Kim, G. H. 838 \& Rhee, S. (2006). Structural and functional analysis of 839 PucM, a hydrolase in the ureide pathway and a 840 member of the transthyretin-related protein family. 841 Proc. Natl Acad. Sci. USA, 103, 9790-9795.

28. Eneqvist, T., Lundberg, E., Karlsson, A., Huang, S., 843 Santos, C. R., Power, D. M. \& Sauer-Eriksson, A. E. 844 (2004). High resolution crystal structures of piscine 845 transthyretin reveal different binding modes for 846 triiodothyronine and thyroxine. J. Biol. Chem. 279, 847 26411-26416.

29. Hennebry, S. C., Law, R. H., Richardson, S. J., Buckle, 849 A. M. \& Whisstock, J. C. (2006). The crystal structure 850 of the transthyretin-like protein from Salmonella 851 dublin, a prokaryote 5-hydroxyisourate hydrolase. 852 J. Mol. Biol. 359, 1389-1399.

30. Otwinowski, Z. \& Minor, W. (1997). Processing of X- 854 ray diffraction data collected in oscillation mode. 855 Methods Enzymol. 276, 307-326.

31. Terwilliger, T. C. \& Berendzen, J. (1999). Automated 85 MAD and MIR structure solution. Acta Crystallogr., 858 Sect. D: Biol. Crystallogr. 55, 849-861.

32. Terwilliger, T. (2004). SOLVE and RESOLVE: auto- 860 mated structure solution, density modification and 861 model building. J. Synchrotron Radiat. 11, 49-52. 862

33. Jones, T. A., Zou, J. Y., Cowan, S. W. \& Kjeldgaard, M. 863 (1991). Improved methods for building protein 864 models in electron density maps and the location of 865 errors in these models. Acta Crystallogr., Sect. A: Found. 866 Crystallogr. 47, 110-119.

(1)


34. Brunger, A. T., Adams, P. D., Clore, G. M., DeLano, W.L., Gros, P., Grosse-Kunstleve, R. W. et al. (1998). Crystallography \& NMR system: a new software suite for macromolecular structure determination. Acta Crystallogr., Sect. D: Biol. Crystallogr. 54, 905-921.

35. McRee, D. E. (1999). XtalView/Xfit-a versatile program for manipulating atomic coordinates and electron density. J. Struct. Biol. 125, 156-165.
36. Brunger, A. T. (1993). Assessment of phase accuracy 876 by cross validation - the free $R$-value. Methods and 877 applications. Acta Crystallogr., Sect. D: Biol. Crystallogr. 878 49, 24-36.

37. Nicholls, A., Sharp, K. A. \& Honig, B. (1991). Protein 880 folding and association: insights from the interfacial 881 and thermodynamic properties of hydrocarbons. 882 Proteins, 11, 281-2961.

876
87
878
879
880
881
882
883

